\title{
Very-high-energy astrophysical neutrinos with IceCube
}

\author{
Ignacio Taboada ${ }^{1, a}$ for the IceCube Collaboration \\ ${ }^{1}$ School of Physics and Center for Relativistic Astrophysics. Georgia Institute of Technology. Atlanta, USA.
}

\begin{abstract}
IceCube is a $\gtrsim \mathrm{TeV}$ neutrino observatory operating at the South Pole. IceCube has observed a flux of neutrinos of astrophysical origin, with energies beyond 2 $\mathrm{PeV}$. However the sources of these neutrinos have not yet been identified. A summary of various IceCube observations is presented. The results discussed were obtained through several different analysis methods, which have varying sensitivity to the different neutrino flavors. A discussion of the spectral fit obtained for the various event selections is included, as well as the constraints on the astrophysical neutrino flavor flux ratio. Several attempts by IceCube to identify the sources of these neutrinos are described. These include studies correlating neutrino events with catalogs of sources as well as selfcorrelations among IceCube's neutrinos. The observations of astrophysical neutrinos are limited by statistics. So an upgrade of IceCube, including a larger detector and a surface veto is planned. This upgrade is briefly discussed.
\end{abstract}

\section{Introduction}

Almost three years ago, IceCube reported the discovery of an astrophysical flux of neutrinos in the $60 \mathrm{TeV}$ to $1 \mathrm{PeV}$ energy range [1]. The observed distribution of neutrinos is consistent with isotropy over the entire $(4 \pi \mathrm{sr})$ sky and there are no obvious individual astrophysical sources responsible for this flux. The discovery study, initially conducted with 2 years of IceCube data has been updated to include another 2 years of data [2,3]. With additional data, the highest energy observed has increased to $\sim 2 \mathrm{PeV}$. The existence of an astrophysical neutrino flux has been verified by several subsequent analyses e.g. Refs. $[4,5]$.

It is a reasonable assumption that cosmic ray sources are also sources of IceCube's neutrinos. It is however possible to explain the neutrino observation assuming that, e.g. dark matter decays into the observed flux. Cosmic rays are charged particles detected at Earth almost isotropically. Cosmic rays are observed in energies from $10^{9} \mathrm{eV}$ to $10^{20} \mathrm{eV}$. Below $\sim 10^{14} \mathrm{eV}$, balloons and satellites observe cosmic rays directly and measure their composition (i.e. the flux proportion of protons, helium, ..., iron). The cosmic ray spectrum is often described by a power law in energy, although there's observational evidence of a large number of features in the spectrum. Direct measurements allow for precise knowledge of the spectra. At higher energies, the steeply falling cosmic ray flux requires the use of ground based detectors. Ground detectors measure the cosmic ray spectra and composition indirectly, via the observation of air showers, which results in less precise measurements. At the highest energies, $>10^{18} \mathrm{eV}$, the Pierre Auger observatory and Telescope Array/HiRes, disagree on the the composition,

\footnotetext{
ae-mail: itaboada@gatech.edu
} 
with the former preferring heavier components (iron-like) and the latter preferring lighter components (proton-like).

There isn't certainty yet on the origin of the cosmic rays. Magnetic confinement of cosmic rays in our Galaxy is expected below the so called cosmic-ray knee $\left(4 \times 10^{15} \mathrm{eV}\right)$, and it is presumed, in this energy range that sources are galactic. The name knee is derived from a softening of the cosmic ray spectrum from an index of $\sim-2.7$ below the knee to $\sim-3$ above the knee. At energies above the ankle $\left(4 \times 10^{18} \mathrm{eV}\right)$ sources are expected to be extra-galactic. It is unclear whether between the knee and the ankle, sources are galactic or extragalactic. For galactic cosmic rays there is evidence that the sources are young, less than a few hundred years old, supernova remnants (SNRs). Motivation for SNRs being the source of galactic cosmic rays is that the mechanical power of all ejecta by supernovae in our galaxy, exceeds the power needed to sustain the cosmic ray population by two orders of magnitude. Shocks in supernova remnants and particle acceleration, such as first order Fermi acceleration, explain the transfer of kinetic energy of the supernova ejecta to cosmic rays.

Sources of cosmic rays are also expected to be sources of $\gamma$-rays and neutrinos. This is because a fraction of the accelerated cosmic rays should interact with material/photon fields at the source or near the source. This interaction results in neutral and charged pions that decay into $\gamma$-rays and neutrinos respectively. Observations of various galactic sources, e.g. Tycho's supernova, SN1572 [6] are consistent with the expectations of cosmic ray production. The young SNR hypothesis is also consistent with the observation of star burst galaxies with $\gamma$-rays [7, 8]. However, it is possible to produce $\mathrm{GeV}$ to $\mathrm{TeV}$ photons without cosmic ray acceleration and therefore observing $\gamma$-rays does not uniquely identify a source as a cosmic ray generator. Furthermore, given a galactic cosmic ray source with emission up to the knee, $\gamma$-rays up to $100 \mathrm{TeV}$ are expected. These observations have not been unequivocally made yet, although there are several candidates, e.g. HESS-J1641-463[9].

Neutrino observations are generally assumed to be uniquely tied to cosmic rays. However as already explained, a diffuse source may have a different explanation. A point-source of neutrinos, however, would be a smoking gun for the observation of a cosmic ray source. IceCube's main science objective, although not the only one, is to detect astrophysical neutrinos and find the sources of cosmic rays.

These proceedings summarize observations by IceCube of the very-high-energy neutrinos of astrophysical origin.

\section{IceCube}

IceCube is a very-high-energy neutrino telescope in operation at the South Pole. Neutrinos are detected by observing secondary particles produced in neutrino-matter interactions. These secondaries produce Cherenkov radiation as they travel in very transparent Antarctic ice. IceCube observes the blue Cherenkov light with a 3-dimensional array of optical sensors (photo-multiplier tubes). The sensors are arranged in the bottom kilometer of vertical strings that are $2.5 \mathrm{~km}$ long. To compensate for the very low neutrino-matter interaction cross-section, the instrumented volume is gigantic: approximately one cubic kilometer for IceCube. A total of 5,160 optical sensors in 86 strings comprise IceCube. There is no sharp energy threshold for neutrino detection in IceCube, with the minimum energy instead, dependent on the selection criteria of each particular analysis. In most studies it is approximately $1 \mathrm{TeV}$. A section of IceCube is instrumented with higher density of optical sensors, allowing for a lower threshold. This subdetector is known as DeepCore. Another subdetector of IceCube is IceTop, an air shower array on the surface which is sensitive to cosmic rays of several PeV and higher. Figure 1 shows IceCube and its subcomponents.

IceCube construction began in 2005 and finished in December 2010. The modular design of IceCube allowed for initial partial operation. In these proceedings the moniker IC-XX represents the 


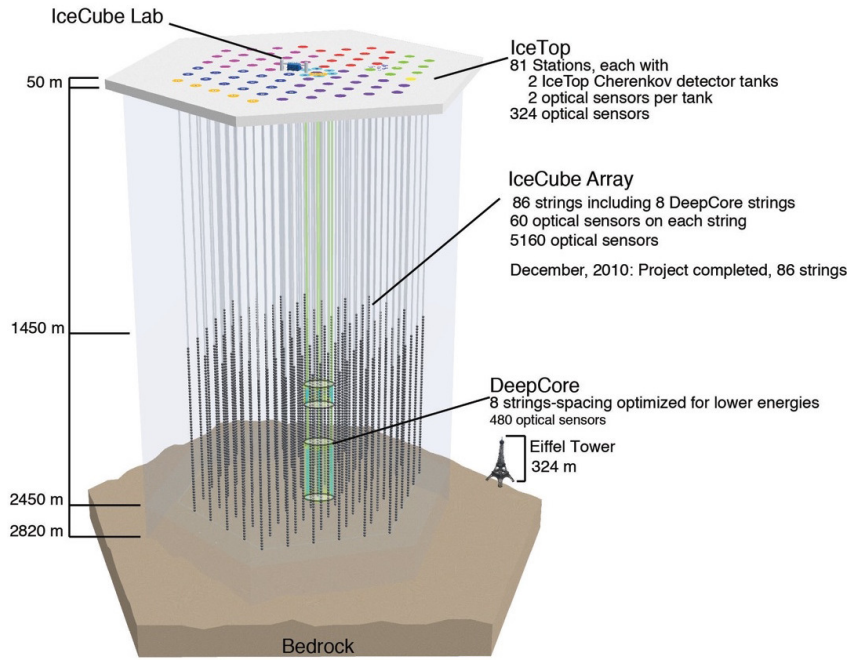

Figure 1. The IceCube neutrino observatory. A total of 86 strings with optical sensors are deployed between 1,450 and $2,450 \mathrm{~m}$ of depth. A subset of strings have been installed closer in horizontal distance and with a higher vertical density of sensors. This subdetector, DeepCore, is located at the center bottom of the rest of IceCube, so that the main array can be used as a muon veto. The surface array, IceTop, consists of 81 pair of ice tanks. Tanks have 2 photomultipliers each, to be sensitive to ground level particles from air showers. Each pair of tanks is located above a string. The detector is located $\sim 1 \mathrm{~km}$ away from the geographical south pole.

number of strings, $\mathrm{XX}$, in a given operational configuration. IceCube operates with extremely high duty cycle, over $99 \%$. And $\sim 99 \%$ of the optical sensors are operational.

There are two main channels for neutrino detection by IceCube. Tracks are made by muons, which are the product of charged current $v_{\mu}$ interactions ${ }^{1}$ with rock or ice near IceCube. A small fraction of the muon channel also arises from $v_{\tau}$ charged current interactions in which the resulting $\tau$ decays into a muon (17.4\% branching ratio). The track channel significantly increases the effective size of the detector since, at $\mathrm{TeV}$ energies and higher the range of muons is in the kilometer scale. The long lever arm of tracks in IceCube allows for good directional reconstruction, from $\sim 1^{\circ}$ at $1 \mathrm{TeV}$ to better than $\sim 0.4^{\circ}$ above hundreds of $\mathrm{TeV}$. Because neutrino interaction vertices are often outside of the detector, the track channel does not measure the neutrino energy directly.

For the shower or cascade channel neutrinos of all flavors are detected via several mechanisms including $\bar{v}_{e}+e^{-}$interactions 6.3 PeV, i.e. the Glashow resonance; $v_{e}$ charged current interactions and neutral current interactions for all flavors. The majority, of $v_{\tau}$ charged current interactions result in cascade channel events. Particle cascades are initiated when the initial neutrino interaction produces particles which subsequently interact through processes such as bremsstrahlung, e-pair production and hadronic interactions resulting in a geometric increase in the number of particles. This increase in particles repeats until a critical energy at which the radiative energy losses are no longer the most significant energy loss mechanism for particles propagating in the ice. A typical shower in ice will have a total length of $\sim 10 \mathrm{~m}$ which is point like compared to the dimensions of IceCube. The lack of lever arm implies that angular resolution is only due to directionality in Cherenkov radiation. This information is partially washed out by optical scattering in the ice. At PeV energies, cascades can be reconstructed to $\sim 10^{\circ}$ and at tens of $\mathrm{TeV}$ to $\sim 30^{\circ}$. On the other hand, cascades are typically detected inside of the detector and all the visible energy is well measured. Furthermore, for most (the exception is the usually sub-dominant neutral current interaction) neutrino interaction channels, the visible energy is well correlated with the neutrino energy. At energies beyond $\sim 500 \mathrm{TeV} v_{\tau}$ interactions can have many complex topologies. However this subject will not be explored further in these proceedings.

\footnotetext{
${ }^{1}$ IceCube is unable to distinguish $v$ from $\bar{v}$
} 
The bulk of measurements, $3 \mathrm{kHz}$, by IceCube is of atmospheric muons, which are produced in cosmic ray air showers. Atmospheric neutrinos are also easily observable with IceCube, at a rate of $\sim 300$ per day with the track channel (exclusively $v_{\mu}$ ) and lower with atmospheric neutrino induced cascades (a mixture of $v_{e}$ and $v_{\mu}$ ). The spectrum of atmospheric muons and neutrinos is softer than that of their parent cosmic rays by approximately one power of energy. Because cosmic rays sources are expected to produce neutrinos with very hard spectra, $d N / d E \propto E^{-2}$, energy is one of the possible characteristics that aids the identification of astrophysical neutrinos.

\section{Discovery of a very-high-energy astrophysical neutrino flux}

Initial evidence for an astrophysical flux was made with the observation of two cascade-like events of PeV energy in a search for ultra-high-energy neutrinos through the Berezinsky-Zatsepin effect, i.e. neutrinos from product of the highest energy cosmic rays, $\sim 10^{19} \mathrm{eV}$, and the cosmic microwave background. These two events [10] did not match the ultra-high-energy neutrino expectation but were too high-energy for the expectations of atmospheric neutrinos.

A follow-up study was designed that focused on events in which the neutrino vertex is contained in a volume slightly less than half that of IceCube. The outer layer of the detector, top and sides, is used to tag down- and through-going events - the signature of atmospheric muons and thus cosmicray air showers. This veto, along with an energy proxy cut (total amount of light observed by the optical sensors) can be used to reduce the background of down-going muons. The energy proxy cut is approximately equivalent to $30 \mathrm{TeV}$. Atmospheric neutrinos are also a background. However downgoing atmospheric neutrinos are vetoed quite efficiently because the air showers that produce them would also produce muons that would be found by the veto.

This method was initially applied to two years of data, one with IC-79 and the first year of IC-86 and was the basis for the initial discovery claim [1]. This study has been repeated for a third [2] and fourth year [3] of data.

The following numbers correspond to the "4-year study", of 1347 days livetime starting on May 2010. The atmospheric background from tagged events is $12.6 \pm 5.1$ events. The atmospheric neutrino background is $9.0_{-2.2}^{+8.0}$. A total of 54 events were found in this study. However posterior examination shows that two are background: one shows coincident activity in IceTop, indicating a simultanous air shower, and the other event corresponds to two almost simultanous but uncorrelated muons, with the first muon disarming the veto and the second one faking a signal. The atmospheric origin of the observations is rejected at the $6.5 \sigma$ level. Of the 52 remaining "good" events, 39 are showers and 13 are tracks - which is consistent with expectations. A sky map with the 52 "good" events is shown in figure 2 .

Using all 54 events, the best fit flux for astrophysical neutrinos is

$$
E^{2} \frac{d N}{d E}=(2.2 \pm 0.7) \times 10^{-8}(E / 100 \mathrm{TeV})^{-0.58 \pm 0.25} \mathrm{GeV}^{-1} \mathrm{~s}^{-1} \mathrm{sr}^{-1} \mathrm{~cm}^{-2}
$$

And this flux is valid in the range $27 \mathrm{TeV}$ to $2 \mathrm{PeV}$. It is also possible to fit the spectrum well including a cutoff, in which case a harder spectral index of -2.31 is found and a cutoff of $2.7 \mathrm{PeV}$ [3].

Southern hemisphere events (down-going) are distributed isotropically, but there is a deficit of northern hemisphere events. This is easily explained by Earth absorption of neutrinos, which is relevant for energies above $\sim 100 \mathrm{TeV}$ and increases with energy. The flux at Earth's surface is consistent with isotropy. 


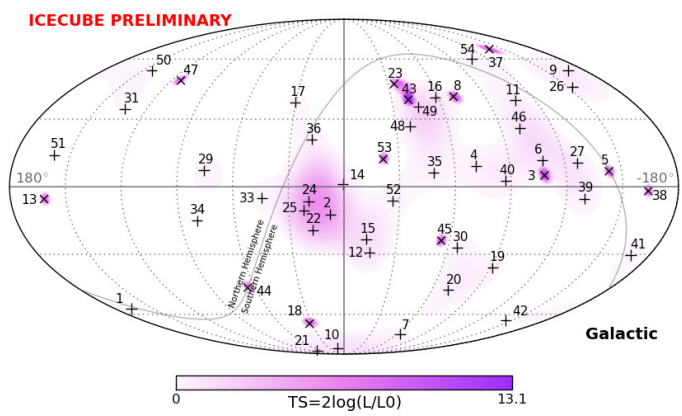

Figure 2. Directions of the 52 candidate astrophysical neutrinos in galactic coordinates. Crosses indicate showerlike events and "X"s show tracks. The color scale is the significance of event excess in a given direction, which depends on the angular uncertainty for each event. Two events are omitted as described in the text. The dashed line shows the northern and southern sky. There is no evidence for a point source in this data set or a correlation with the galactic plane.

\section{Confirmation with muon neutrinos}

The traditional neutrino astronomy channel is the track channel in which Earth acts as a filter by blocking up-going (nothern sky) atmospheric muons and only atmospheric $v_{\mu}$ remain as a significant background. This background can be further reduced via energy or energy-proxies. The most recent study by IceCube is for " 6 years" including IC-40, IC-59, IC-79 ( $\approx 1$ year each) and 3 years of IC-86 [3]. The astrophysical flux is also identified in this study with a best fit spectrum:

$$
E^{2} \frac{d N}{d E}=0.78_{-0.25}^{0.29} \times 10^{-8}(E / 100 \mathrm{TeV})^{-0.6 \pm 0.13} \mathrm{GeV}^{-1} \mathrm{~s}^{-1} \mathrm{sr}^{-1} \mathrm{~cm}^{-2}
$$

which is valid for the energy range $160 \mathrm{TeV}$ to $10 \mathrm{PeV}$. The atmospheric only hypothesis is rejected at the $6.0 \sigma$ level. Figure 3 shows the energy spectrum of events as found by this study. The flux is also consistent with the expectation of isotropically distributed sources - although in this case the sensitivity of the analysis is limited to declinations between -5 to $90^{\circ}$.

Other studies have been conducted by IceCube that are also sensitive to the astrophysical flux $[5,11]$. In particular, the starting event method used for discovery has been used with an energydependent veto region that allows the extension of observations to as low as $10 \mathrm{TeV}$. In this case the spectrum seems to be well described by a power law with index -2.49 . There is an apparent discrepancy in spectrum as measured with starting events and with tracks. It should be noted that the sensitivity in energy range of both methods overlap but are not identical. The limited statistics in number of astrophysical events is such that inconsistent power law fits can describe the data of each method well, even if the true flux has a more complicated description than a power law. It is possible that two or more populations of sources produce the observed flux. However the statistics necessary to test this hypothesis are beyond the reach of IceCube.

\section{The search for sources of the very-high-energy neutrinos}

A very large number of explanations for the origin of IceCube's astrophysical flux have been offered in the literature. Consistency with isotropy for the observations obtained with both methods described 


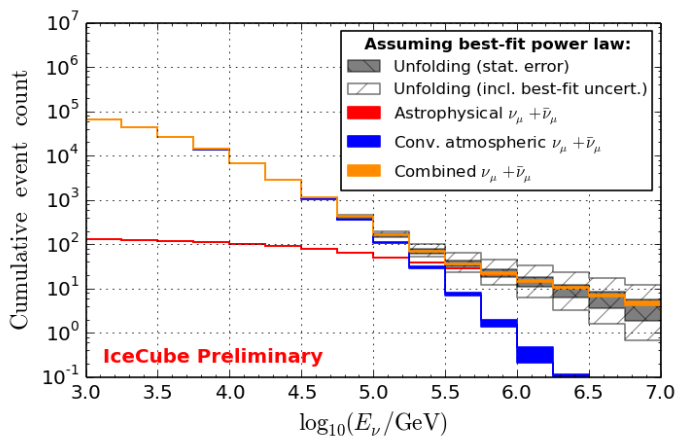

Figure 3. Energy spectrum of up-going $v_{\mu}$ event candidates. The red line corresponds to the best fit astrophysical flux assuming a power law in energy. Atmospheric neutrino background is shown in blue.

indicates a dominant extragalactic origin. The data in both cases has been studied for correlation with the galactic plane. No significant correlation is found in any of the analysis. For the starting event method used for discovery, the best correlation is for $\pm 2.5^{\circ}$ above and below the galactic plane with p-value of 7\%. This indicates that the contribution from objects in the galactic plane is not dominant.

Searches for self-correlation in the starting event sample fail to find one or more point sources of neutrinos. With an event selection similar to that used for the track study described in section 4 , a large collection of $v_{\mu}$ events has been accumulated of a period of 6 years. A self-correlation study on directions with this data set also fails to find point sources. This latter study is so sensitive that if the astrophysical flux were due to a handful of sources, they would have already been detected as point sources. This lack of observations in point sources places a limit on the number of (neutrino) visible sources to $>100$ over the entire sky ( $4 \pi$ sr) [2].

IceCube has searched, with null results, for neutrinos in correlation with over 1000 gamma ray bursts (GRBs) reported by various satellites. For GRBs, it is assumed that neutrinos are produced simultanously with the $\mathrm{keV}-\mathrm{MeV}$ photons, the prompt phase. IceCube studies conclude that during prompt phase, at most 1-3\% of the observed astrophysical flux is due to GRBs [14]. Even if the correlation time is allowed to expand, GRBs cannot produce more than $12 \%$ of the spectrum for times of \pm 20 hours around GRBs.

Active galactic nuclei are another prominent candidate for cosmic ray sources [15]. A study searching for correlation with a stacked list of blazars detected by Fermi-LAT, suggests that less than $30 \%$ of the observed flux originates from these. This limit however is quite model dependent given the strong time variability of blazars. Finally a study on the diffuse extragalactic background as observed by Fermi-LAT, rules out star-burst galaxies as the major component of the neutrino astrophysical flux [16].

So the most popular candidates, GRBs, AGNs and star-burst galaxies are constrained or ruled out. The question of the origin of the astrophysical neutrinos remains open.

It is interesting to study the flavor flux ratio of neutrinos. In the most naive interpretation, neutrinos are expected to be produced at cosmic ray sources with a flavor flux ratio of 1:2:0 $\left(f_{v_{e}}: f_{v_{\mu}}: f_{v_{\tau}}\right)$. And vacuum oscillations over astronomical baselines are such that a ratio of 1:1:1 is expected at Earth. This expectation can be modified, e.g. assuming strong magnetic fields at the sources that cool down some of the particles (muons) that produce neutrinos leading to a ratio of 0.4:0.6:0.6 at Earth [12]. Both the shower and the track channels are sensitive to mixtures of the three flavors. IceCube has 
conducted a study [13] in which various observations of the astrophysical flux are studied jointly. It is found that IceCube's data is consistent with all expected astrophysical flavor flux ratios. This can be seen in figure 4 .

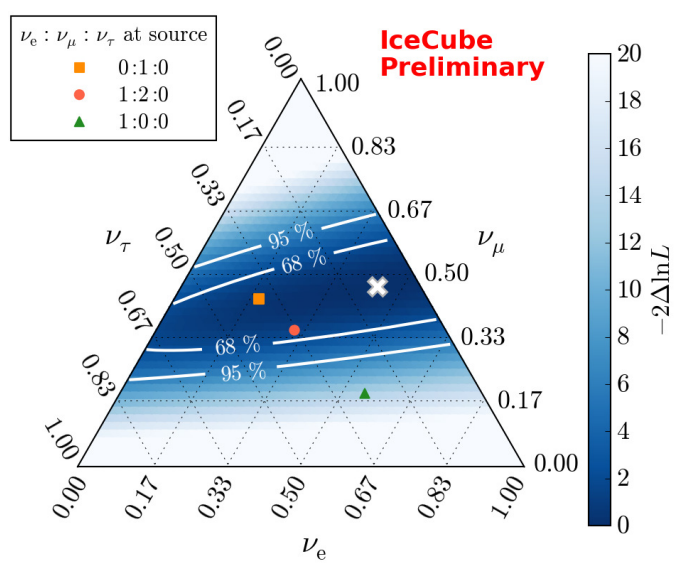

Figure 4. Flavor triangle plot, all three flavor components are free to float in a global fit of IceCube observations, but assumed to all follow the same energy spectrum. In this case spectral index, flux normalization and cutoff are fitted. The standard astrophysical assumption 1:2:0 at the source. Pure flavor composition, of any type, are ruled out.

\section{Conclusions and Outlook}

IceCube continues to collect that with very high uptime, stability and quality. The astrophysical flux has been observed with various methods with a combined sensitivity to 3 orders of magnitude in energy. The observed spectrum appears consistent with a dominant extragalactic origin, but the most well known candidates, GRBs, AGNs and star burst galaxies have been constrained or ruled out.

IceCube is proposing the construction of IceCube Gen2, which would consist of a larger underice component as well as a large area surface detector to act as a very wide angle veto.

\section{References}

[1] Aarsten M.G. et al. (IceCube collaboration), Science 342, 6161 (2013)

[2] Aartsen M.G. et al. (IceCube collaboration), Phys. Rev. Lett. 113, 101101 (2014)

[3] Aarsten M.G. et al. (IceCube collaboration), Proc. of the 34th Int. Cosmic Ray Conf. arXiv:1510.05223, The Hague (2015).

[4] Aartsen M.G. et al. (IceCube collaboration), Phys. Rev. Lett. 115, 081102 (2015)

[5] Aartsen et al. M.G. (IceCube collaboration) Phys. Rev. D91, 022001 (2015)

[6] Acciari V.A. et al. (VERITAS collaboration), ApJ 730, L20 (2011)

[7] Acciari V.A. et al. (VERITAS collaboration), Nature 462, 770 (2009)

[8] Abdo A.A. (Fermi collaboration), ApJ 709, L152 (2010)

[9] Abramowski A. et al. (HESS collaboration) ApJ 794, L1 (2014) 
[10] Aarsten M.G. et al. (IceCube collaboration) Phys. Rev. Lett. 111, 021103 (2013).

[11] Aarsten et al. M.G. (IceCube collaboration), ApJ 809, 98 (2015)

[12] Kashti T. Waxman E., Phys. Rev. Lett. 95, 181101 (2005)

[13] Aartsen M.G. et al. (IceCube collaboration), Phys. Rev. Lett. 114, 171102 (2015).

[14] Aartsen M.G. et al. (IceCube collaboration), ApJ 805, L5 (2015)

[15] Padovani P., Petropoulou M., Giommi P. and Resconi E. Submitted to MNRAS. arXiv:1506.09135

[16] Bechtol K., Ahlers M., Di Mauro M, Ajello M and Vandenbroucke J. Submitted to Phys. Rev. Lett. arXiv: 1511.00688 